\title{
Relating invariant linear form and local epsilon factors via global methods
}

\author{
Dipendra Prasad
}

\begin{abstract}
We use the recent proof of Jacquet's conjecture due to Harris and Kudla, and the Burger-Sarnak principle to give a proof about the relationship between the existence of trilinear forms on representations of $G L_{2}\left(k_{u}\right)$ for a non-Archimedean local field $k_{u}$ and local epsilon factors which was earlier proved only in the odd residue characteristic by this author in [P1]. The same method gives a global proof of a theorem of Saito and Tunnell about characters of $G L_{2}$ using a theorem of Waldspurger about period integrals for $G L_{2}$ and also an extension of the theorem of Saito-Tunnell by this author in [P2] which was earlier proved only in odd residue characteristic.
\end{abstract}

\section{Triple products}

Let $\pi_{1}, \pi_{2}$ and $\pi_{3}$ be three irreducible admissible infinite dimensional representations of $G L_{2}\left(k_{u}\right)$ for a non-Archimedean local field $k_{u}$ with the product of their central characters trivial. Let $D_{u}$ denote the unique quaternion division algebra over $k_{u}$. For an irreducible admissible discrete series representation $\pi$ of $G L_{2}\left(k_{u}\right)$, let $\pi^{\prime}$ denote the representation of $D_{u}^{*}$ associated to $\pi$ by the Jacquet-Langlands correspondence, and let $\pi^{\prime}=0$, if $\pi$ is not a discrete series representation.

The author in his thesis [P1] studied the space of trilinear forms $\ell: \pi_{1} \otimes$ $\pi_{2} \otimes \pi_{3} \rightarrow \mathbb{C}$ which are $G L_{2}\left(k_{u}\right)$-invariant. Let $m\left(\pi_{1} \otimes \pi_{2} \otimes \pi_{3}\right)$ denote the dimension of the space of such trilinear forms, and let $m\left(\pi_{1}^{\prime} \otimes \pi_{2}^{\prime} \otimes \pi_{3}^{\prime}\right)$ denote the dimension of the space of $D_{u}^{*}$-invariant linear forms on $\pi_{1}^{\prime} \otimes \pi_{2}^{\prime} \otimes \pi_{3}^{\prime}$ (so $m\left(\pi_{1}^{\prime} \otimes \pi_{2}^{\prime} \otimes \pi_{3}^{\prime}\right)$ is nonzero only if all the $\pi_{i}$ are discrete series representations). We let $\epsilon\left(\pi_{1} \otimes \pi_{2} \otimes \pi_{3}\right)=\epsilon\left(\frac{1}{2}, \pi_{1} \otimes \pi_{2} \otimes \pi_{3}\right)$ denote the triple product epsilon factor defined by the Langlands-Shahidi method; under the condition that the 
product of the central characters is trivial, $\epsilon\left(\pi_{1} \otimes \pi_{2} \otimes \pi_{3}\right)= \pm 1$. Usually, the epsilon factor depends on an auxiliary additive character of the field, but in our case it is independent of it.

The main results proved in $[\mathrm{P} 1]$ were the following.

1. Multiplicity one theorem: $m\left(\pi_{1} \otimes \pi_{2} \otimes \pi_{3}\right) \leq 1$.

2. Dichotomy principle: $m\left(\pi_{1} \otimes \pi_{2} \otimes \pi_{3}\right)+m\left(\pi_{1}^{\prime} \otimes \pi_{2}^{\prime} \otimes \pi_{3}^{\prime}\right)=1$.

3. Theorem about epsilon factors: $m\left(\pi_{1} \otimes \pi_{2} \otimes \pi_{3}\right)=1$ if and only if $\epsilon\left(\pi_{1} \otimes \pi_{2} \otimes \pi_{3}\right)=1$.

A few words about the proofs. The multiplicity one theorem was proved by the method of Gelfand pairs, as developed by Gelfand and Kazhdan, and was thus based on general principles available to prove such theorems.

The dichotomy principle was eventually related to the character identity

$$
\Theta_{\pi}(x)=-\Theta_{\pi^{\prime}}(x)
$$

at regular elliptic elements $x$ for discrete series representations $\pi$ and $\pi^{\prime}$ of $G L_{2}\left(k_{u}\right)$ and $D_{u}^{*}$ respectively, associated to each other by the Jacquet-Langlands correspondence; this combined with the theorem on finite groups according to which

$$
m\left(\pi_{1}^{\prime} \otimes \pi_{2}^{\prime} \otimes \pi_{3}^{\prime}\right)=\frac{1}{\operatorname{vol}\left(D_{u}^{*} / k_{u}^{*}\right)} \int_{D_{u}^{*} / k_{u}^{*}} \Theta_{\pi_{1}^{\prime}}(g) \Theta_{\pi_{2}^{\prime}}(g) \Theta_{\pi_{3}^{\prime}}(g) d g,
$$

and a suitable variant for $G L_{2}\left(k_{u}\right)(!)$, proves the dichotomy principle for supercuspidal representations, others being much more straightforward by the orbit method of Mackey.

These two theorems had reasonably satisfactory proofs. However, the theorem about epsilon factors was proved in a case-by-case way by reducing it to simpler epsilon factors studied by Tunnell [T], and this reduction was possible only for representations of $G L_{2}\left(k_{u}\right)$ arising from characters of quadratic extensions of $k_{u}$, and was thus incomplete in even residue characteristic. Also, the proof of the theorem on epsilon factors given in [P1] left much to be desired as it was brute force calculation which essentially amounted to calculating epsilon factor of the triple product and relating it to $m\left(\pi_{1} \otimes \pi_{2} \otimes \pi_{3}\right)$ through an explicit knowledge of the character of representations of $G L_{2}\left(k_{u}\right)$ which has been known for a long time through the work of Sally and Shalika, the epsilon factor 
(associated to Galois representations) being calculated either directly, or through Tunnell's work which also was a brute force calculation with characters. (Later, there was the elegant paper of Saito [Sa] which proved Tunnell's theorem in all residue characteristics.)

The aim of this paper is to offer a global method for the result on epsilon factors using the fact that the global analogue of the results on trilinear form, which is the period integral, has recently been proved by Harris and Kudla (this result was conjectured by $\mathrm{H}$. Jacquet). More importantly, the present proof, unlike the earlier one, offers a conceptual reason why the theorem on epsilon factors holds good, and suggests that the natural proof of the general conjectures of Gross and the author in [G-P] about local branching laws from $S O(n)$ to $S O(n-1)$ in terms of epsilon factors may consist of the following 3 steps.

1. Proof of the global conjecture in [G-P] about nonvanishing of the period integral in terms of $L$-value at $1 / 2$.

2. Proof of the conjecture about branching from $S O(n)$ to $S O(n-1)$ for unramified principal series representations of $S O(n)$.

3. Proof of the conjecture for $\mathbb{R}$.

In the cases studied in this paper which consists of cases of $n \leq 4$, all the 3 steps are known. However, for general n, none of the 3 steps are known at present.

We now recall the theorem of Harris and Kudla.

Theorem 1 (Harris-Kudla) Let $k$ be a number field, and $\Pi_{1}, \Pi_{2}$ and $\Pi_{3}$ three cuspidal automorphic representations of $G L_{2}\left(\mathbb{A}_{k}\right)$ with the product of their central characters trivial. For a quaternion division algebra $D$ over $k$, let $\Pi_{i}^{D}$ be the automorphic representations of $\left(D \otimes_{k} \mathbb{A}_{k}\right)^{*}$ associated to $\Pi_{i}$ by the global Jacquet-Langlands correspondence, if it exists. Then the central critical L-value $L\left(\frac{1}{2}, \Pi_{1} \otimes \Pi_{2} \otimes \Pi_{3}\right)$ is nonzero if and only if for some $D, \Pi_{i}^{D}$ exist as automorphic representations for which there are $f_{i}^{D} \in \Pi_{i}^{D}$ such that

$$
\int_{D^{*} \mathbb{A}_{k}^{*} \backslash\left(D \otimes_{k} \mathbb{A}_{k}\right)^{*}} f_{1}^{D}(g) f_{2}^{D}(g) f_{3}^{D}(g) d^{\times} g \neq 0 .
$$

The proof of the above theorem does not use the theorem on epsilon factors, so it is legitimate to use it to prove the following theorem. 
Theorem 2 Let $\pi_{1}, \pi_{2}$ and $\pi_{3}$ be three irreducible admissible infinite dimensional representations of $G L_{2}\left(k_{u}\right)$ for a non-Archimedean local field $k_{u}$ with the product of their central characters trivial. Then there exists a nonzero $\ell: \pi_{1} \otimes \pi_{2} \otimes \pi_{3} \rightarrow \mathbb{C}$ which is $G L_{2}\left(k_{u}\right)$-invariant if and only if $\epsilon\left(\pi_{1} \otimes \pi_{2} \otimes \pi_{3}\right)=1$.

Proof: If one of the representations $\pi_{i}$ is either principal series, or is a twist of the Steinberg representation, then the result can be proved by simple calculations as done in [P1]. (Essentially because in all these cases except when one is dealing with the triple product of the Steinberg, there is an invariant linear form as follows by simple orbit methods; also, the epsilon factor is easily calculated to be one in all these cases.) We will, therefore, in the rest of the proof assume that all the representations $\pi_{i}$ are supercuspidal representations of $G L_{2}\left(k_{u}\right)$ with the product of their central characters trivial.

We will first prove that if there is a nonzero $\ell: \pi_{1} \otimes \pi_{2} \otimes \pi_{3} \rightarrow \mathbb{C}$ which is $G L_{2}\left(k_{u}\right)$-invariant, then $\epsilon\left(\pi_{1} \otimes \pi_{2} \otimes \pi_{3}\right)=1$. Then we will use the dichotomy principle to say that if the space of $G L_{2}\left(k_{u}\right)$-invariant forms on $\pi_{1} \otimes \pi_{2} \otimes \pi_{3}$ is zero, then $\pi_{i}$ are all discrete series representations, and for the corresponding representations $\pi_{i}^{\prime}$ of $D_{u}^{*}$, there is a $D_{u}^{*}$-invariant linear form on $\pi_{1}^{\prime} \otimes \pi_{2}^{\prime} \otimes \pi_{3}^{\prime}$. Now by the same method employed to prove the first case (when there is a $G L_{2}\left(k_{u}\right)$ invariant linear form), we prove that the local epsilon factor $\epsilon\left(\pi_{1} \otimes \pi_{2} \otimes \pi_{3}\right)$ in this case is -1 . This will complete the proof of the theorem.

Fix a totally real number field $k$ and a place $u$ of $k$ such that the completion of $k$ at $u$ is the local field $k_{u}$ that we started with. Let $D$ be a quaternion division algebra over $k$ which is unramified at all the finite places, and for simplicity, we assume that $D$ is ramified at all the infinite places. This can be done by choosing $k$ appropriately so that it has even degree over $\mathbb{Q}$.

We let $\Pi_{1}$ and $\Pi_{2}$ be automorphic representations of $D\left(\mathbb{A}_{k}\right)^{*}$ with local components $\pi_{1}$ and $\pi_{2}$ respectively at $u$, unramified at all the other finite places outside $u$. It is well known that local supercuspidal representations can be obtained as the local component of an automorphic representation which is unramified at all the other finite places, and have some weights at infinity.

We will choose $\Pi_{3}$ which will have $\pi_{3}$ as its local component at $u$ (and some infinity type). The representation $\Pi_{3}$ will be so constructed that the period integral

$$
\int_{D^{*} \mathbb{A}_{k}^{*} \backslash D^{*}\left(\mathbb{A}_{k}\right)} f_{1}(g) f_{3}(g) f_{3}(g) d^{\times} g,
$$

is nonzero for some choice of functions $f_{i} \in \Pi_{i} \subset L^{2}\left(D^{*} \backslash D^{*}\left(\mathbb{A}_{k}\right)\right)$. We will 
state the general lemma below, which is part of Burger-Sarnak philosophy, which implies that such choices can be made.

Once we have automorphic representations $\Pi_{i}$ with nonvanishing period integral, the theorem of Harris and Kudla implies that

$$
L\left(\frac{1}{2}, \Pi_{1} \otimes \Pi_{2} \otimes \Pi_{3}\right) \neq 0 .
$$

This implies, in particular, that the global sign, $\epsilon\left(\frac{1}{2}, \Pi_{1} \otimes \Pi_{2} \otimes \Pi_{3}\right)$, in the functional equation for $L\left(s, \Pi_{1} \otimes \Pi_{2} \otimes \Pi_{3}\right)$ is 1 . However, the global sign in the functional equation is nothing but the product of the local epsilon factors. From the information that $\Pi_{1}$ is a principal series representation at all the finite places of $k$ except $u$ it is easy to see that the epsilon factor at all the finite places except $u$ is 1: this just follows from the general fact that $\epsilon(\sigma) \epsilon\left(\sigma^{\vee}\right)=\operatorname{det}(\sigma)(-1)$.

The triple product epsilon factor at all the infinite places is -1 by invoking the corresponding theorem at infinity (the local representations at infinity are given to have invariant linear forms); these calculations at infinity are simple consequences of results about the decomposition of the tensor product of finite dimensional representations of $D^{*}(\mathbb{R})$, the so called Clebsch-Gordon theorem, and are discussed in section 9 of [P1].

It is clear then from the factorisation

$$
\epsilon\left(\frac{1}{2}, \Pi_{1} \otimes \Pi_{2} \otimes \Pi_{3}\right)=\prod_{\mu} \epsilon\left(\frac{1}{2}, \Pi_{1, \mu} \otimes \Pi_{2, \mu} \otimes \Pi_{3, \mu}\right)=1,
$$

and recalling that there are an even number of places at infinity that $\epsilon\left(\pi_{1} \otimes\right.$ $\left.\pi_{2} \otimes \pi_{3}\right)=1$, thus proving that if the space of $G L_{2}\left(k_{u}\right)$-invariant linear forms on $\pi_{1} \otimes \pi_{2} \otimes \pi_{3}$ is nonzero, the triple product epsilon factor $\epsilon\left(\pi_{1} \otimes \pi_{2} \otimes \pi_{3}\right)$ is 1 .

Now, by the Dichotomy theorem, when there is no $G L_{2}\left(k_{u}\right)$-invariant trilinear form, there will be one on the corresponding representations $\pi_{i}^{\prime}$ of $D_{u}^{*}$, and we can do exactly the same analysis by choosing a quaternion division algebra over $k$ which is ramified exactly at $u$, and at all the infinite places of $k$ which are now assumed by choosing the number field $k$ appropriately to be odd in number. Once again the sign in the functional equation will be one, and since the theorem at infinity gives the sign -1 at each of the infinite places, we get in this case $\epsilon\left(\pi_{1} \otimes \pi_{2} \otimes \pi_{3}\right)=-1$, completing the proof of the theorem. Le us emphasize that in our proof the local epsilon factor at a finite place is matched to one at infinity by global means, reducing us to a much simpler problem. 
Remark : The epsilon factor used in [P1] were those arising from Galois representations, whereas the epsilon factors used here are those defined by the Langlands-Shahidi method. It is a theorem of Ramakrishnan, cf. theorem 4.4.1 of [Ra], that these two epsilon factors are the same.

The following result is essentially due to Burger and Sarnak [BS], but not quite! A result of this kind only for the infinite prime is Proposition 3.1 of $[\mathrm{HL}]$. Adding finite primes causes no extra difficulty. However, for the sake of completeness we give a self-contained proof of a result which is adequate for our purposes, and which is totally transparent and elementary consequence of the weak approximation theorem.

Lemma 1 Let $k$ be a number field, $S$ a finite set of finite places of $k, G$ a reductive algebraic group defined over $k$, and $H$ a reductive subgroup of $G$. Suppose that $Z$ is a central subgroup of $H$, which remains central in $G$ with the property that $Z \backslash H$ has no nontrivial k-rational characters. Let $G_{S}=\prod_{v \in S} G\left(k_{v}\right)$, similarly let $H_{S}=\prod_{v \in S} H\left(k_{v}\right)$. Let $\pi=\otimes_{v} \pi_{v}$ be an automorphic representation of $G\left(\mathbb{A}_{k}\right)$. Suppose that $\mu_{v}$ are supercuspidal representations of $H\left(k_{v}\right)$, $v \in S$, which are induced from representations $\nu_{v}$ of subgroups $\mathcal{K}_{v}$ which are certain open subgroups of $H_{v}$, compact modulo $Z_{v}$. Assume that $\mu_{v}$ appears as a quotient of $\pi_{v}$ restricted to $H\left(k_{v}\right)$ for all $v \in S$. Then there is an automorphic representation $\mu=\otimes_{v} \mu_{v}$ of $H\left(\mathbb{A}_{k}\right)$ with $\mu_{S}=\otimes_{v \in S} \mu_{v}$, and functions $f_{1} \in \pi$, $f_{2} \in \mu$ such that

$$
\int_{H(k) Z\left(\mathbb{A}_{k}\right) \backslash H\left(\mathbb{A}_{k}\right)} f_{1} \bar{f}_{2} d h \neq 0 .
$$

Proof : By the assumption that $\mu_{v}$ appears as a quotient of $\pi_{v}$ restricted to $H\left(k_{v}\right)$ for all $v \in S$, the representation $\nu_{v}$ of $\mathcal{K}_{v}$ is a subrepresentation of $\pi_{v}$ restricted to $\mathcal{K}_{v}$ for all $v \in S$. This means that there is a function $f$ on $G(k) \backslash G\left(\mathbb{A}_{k}\right)$ whose $\mathcal{K}_{S}=\prod_{v \in S} \mathcal{K}_{v}$ translates generate a space of functions which is isomorphic to $\otimes \nu_{v}$ as $\mathcal{K}_{S}$-modules. We prove that the restriction of one such function to $H(k) \backslash H\left(\mathbb{A}_{k}\right)$ is not identically zero. Observe that $G\left(\mathbb{A}^{S}\right)=$ $\left\{x \in G(\mathbb{A}), x=\prod_{v} x_{v} \mid x_{v}=1, \forall v \in S\right\}$ operates on such functions (by right translation), and if all the $G\left(\mathbb{A}^{S}\right)$ translates of a function $f$ were zero at the identity element of $G(\mathbb{A})$, the function $f$ would be identically zero by the weak approximation theorem according to which $G(k)$ is dense in $G_{S}$. Thus we have a function $f$ on $G(k) \backslash G\left(\mathbb{A}_{k}\right)$ whose restriction to $H(k) \backslash H\left(\mathbb{A}_{k}\right)$, say $\tilde{f}$, is not zero. The $\mathcal{K}_{S}$ translates of $\tilde{f}$ generates a space of functions now on $H(k) \backslash H\left(\mathbb{A}_{k}\right)$ 
which is isomorphic to $\otimes \nu_{v}$ as $\mathcal{K}_{S}$-modules. Since,

$$
\mu_{S}=\operatorname{ind}_{\mathcal{K}_{S}}^{H_{S}} \nu_{S}
$$

the $H_{S}$-translates of $\tilde{f}$ generates a space of functions on $H(k) \backslash H\left(\mathbb{A}_{k}\right)$ which is isomorphic to $\mu_{S}$ as $H_{S}$-modules. We are now done by the next lemma.

Lemma 2 Suppose that $H$ is a reductive algebraic group over a number field $k$ with center $Z$. Suppose that $S$ is a finite set of finite places and $H_{S}=$ $\prod_{v \in S} H\left(k_{v}\right)$. Suppose that $\tilde{f}$ is a continuous function on $H(k) \backslash H\left(\mathbb{A}_{k}\right)$ with unitary central character $\chi$ whose $H_{S}$ translates generate an irreducible $H_{S^{-}}$ submodule, say $\mu_{S}$, of a space of functions on $H(k) \backslash H\left(\mathbb{A}_{k}\right)$. Then there exists an automorphic function $g$ on $H\left(\mathbb{A}_{k}\right)$ with unitary central character $\chi$ generating an irreducible representation with $H_{S}$-type $\mu_{S}$, and with

$$
\int_{H(k) Z\left(\mathbb{A}_{k}\right) \backslash H\left(\mathbb{A}_{k}\right)} \tilde{f} \bar{g} d h \neq 0 .
$$

Proof : The proof of the lemma follows from the fact that the space of automorphic forms on $H\left(\mathbb{A}_{k}\right)$ is dense in the space of continuous functions. The nonvanishing of the integral implies that the $H_{S}$-type of the space generated by $g$ is the same as that of the space generated by $\tilde{f}$ which is $\mu_{S}$.

Remark : In the proof of theorem 2, we apply lemma 1 to $\mathrm{G}=G L_{2} \times G L_{2}, H=$ $G L_{2}$, and $Z=G_{m}$, using the well-known theorem of Kutzko that a supercuspidal representation of $G L_{2}\left(k_{u}\right)$ can be obtained as an induced representation from a finite dimensional representation of an open subgroup which is compact modulo center.

\section{Saito-Tunnell}

By exactly the same method as employed in the previous section, one can deduce the theorem of Saito and Tunnell which describes which characters of $L^{*}$, for $L$ a quadratic extension of a local field $k_{u}$, appear in an irreducible admissible infinite dimensional representations of $G L_{2}\left(k_{u}\right)$, or in an irreducible representation of $D_{u}^{*}$, where $D_{u}$ is the unique quaternion division algebra over $k_{u}$, in terms of the local epsilon factors. It is elementary to see that characters of $L^{*}$ appear 
with multiplicity at most 1 in any irreducible representation of $G L_{2}\left(k_{u}\right)$, or of $D_{u}^{*}$; see for example, remark 3.5 of [P1]. The dichotomy principle too holds in this situation, i.e., if $\pi$ is a discrete series representation of $G L_{2}(k)$, and $\pi^{\prime}$ is the corresponding finite dimensional irreducible representation of $D_{u}^{*}$, then for a character $\chi$ of $L^{*}$ whose restriction to $k_{u}^{*}$ is the same as the central character of $\pi, \chi$ appears in exactly one of the representations $\pi$ or $\pi^{\prime}$. This follows from the character identity

$$
\Theta_{\pi}(x)=-\Theta_{\pi^{\prime}}(x) .
$$

By appealing to a global theorem due to Waldspurger, we give a proof of the Saito-Tunnell theorem. However, there seems little point in giving many details except to recall the statements of the theorems.

Theorem 3 (Waldspurger) Let $k$ be a number field, $F$ a quadratic extension of $k, D$ a quaternion division algebra over $k$ containing $F$, and $\pi^{\prime}$ an automorphic form on $D(\mathbb{A})^{*}$ realised on a space of functions $E^{\prime}$ on $D^{*} \backslash D\left(\mathbb{A}_{k}\right)^{*}$. Let $T$ be the torus inside $D^{*}$ defined by $F$, and $\Omega=\otimes \Omega_{v}$ a continuous character of $T(k) \backslash T\left(\mathbb{A}_{k}\right)$ whose restriction to the center of $D(\mathbb{A})^{*}$ is the same as the central character of $\pi^{\prime}$. Then there exists a function $e^{\prime}$ in $E^{\prime}$ such that the integral

$$
\int_{T(k) \mathbb{A}_{k}^{*} \backslash T\left(\mathbb{A}_{k}\right)} e^{\prime}(t) \Omega^{-1}(t) d t,
$$

is nonzero if and only if the following two conditions are verified:

1. For all places $v$ of $k$, the local representation $\pi_{v}^{\prime}$ has $\Omega_{v}$-invariant linear form for the torus $\left(F \otimes k_{v}\right)^{*}$.

2. If $\Pi$ denotes the base change of $\pi$ to $G L_{2}\left(\mathbb{A}_{F}\right)$,

$$
L\left(\frac{1}{2}, \Pi \otimes \Omega^{-1}\right) \neq 0 .
$$

We now state the theorem of Saito and Tunnell which follows from the theorem of Waldspurger just as our earlier proof. In this theorem, the epsilon factor is sensitive to the additive character chosen. The theorem is deduced from the theorem of Waldspurger which has no reference to the additive character. The reason for this of course is that the corresponding statement at infinity also depends on the choice of such an additive character. (Note that fixing a character of $\mathbb{A}_{k} / k$ at one place of $k$ fixes it also at any other place of $k$ because $k_{v}$ is dense 
in $\mathbb{A}_{k} / k$ for any place $v$ of $k$.) The theorem of Saito and Tunnell although not stated in their papers for Archimedean fields is valid for such fields too, and has quite an elementary proof.

Theorem 4 (Saito, Tunnell) Let $\pi$ be an infinite dimensional irreducible admissible representation of $G L_{2}\left(k_{u}\right), L$ a quadratic extension of $k_{u}$, and $\Pi$ the base change lift of $\pi$ to $G L_{2}(L)$. Fix a nontrivial additive character $\psi$ of $L$ which restricted to $k$ is trivial. Then for a character $\Omega_{u}$ of $L^{*}$ which has the same restriction to $k_{u}^{*}$ as the central character of $\pi, \epsilon\left(\Pi \otimes \Omega_{u}^{-1}, \psi\right)$ is indepenent of $\psi$ (as long as its restriction to $k_{u}$ is trivial), and takes the value \pm 1 . The character $\Omega_{u}$ of $L^{*}$ appears in $\pi$ if and only if

$$
\epsilon\left(\Pi \otimes \Omega_{u}^{-1}, \psi\right)=1
$$

\section{Extending Saito-Tunnell}

Let $L$ be a quadratic extension of a local field $k_{u}$ of characteristic $\neq 2$. The theorem of Saito and Tunnell discussed in the previous section describes which characters of $L^{*}$ appear in an irreducible admissible infinite dimensional representations of $G L_{2}\left(k_{u}\right)$, or in an irreducible representation of $D_{u}^{*}$ where $D_{u}$ is the unique quaternion division algebra over $k_{u}$ in terms of the local epsilon factors. If the representation $\pi_{\theta}$ of $G L_{2}\left(k_{u}\right)$ comes from a character $\theta$ of $L^{*}$ via the construction of the Weil representation, cf. [J-L, theorem 4.6], the representation $\pi_{\theta}$ decomposes into two irreducible representations $\pi_{\theta}=\pi_{+} \oplus \pi_{-}$when restricted to $G L_{2}\left(k_{u}\right)^{+}=\left\{x \in G L_{2}\left(k_{u}\right) \mid \operatorname{det}(x) \in N L^{*}\right\}$ where $N L^{*}$ is the subgroup of $k_{u}^{*}$ of index 2 consisting of norms from $L^{*}$; similarly one can define a subgroup of index 2 inside $D_{u}^{*}$, to be denoted by $D_{u}^{*+}$, and for which one has a similar decomposition $\pi_{\theta}^{\prime}=\pi_{+}^{\prime} \oplus \pi_{-}^{\prime}$ of the corresponding representation of $D_{u}^{*}$. Clearly $L^{*}$ is contained in both $G L_{2}\left(k_{u}\right)^{+}$, and $D_{u}^{*+}$, and one can ask about a generalisation of the theorem of Saito and Tunnell to describe the decomposition of $\pi_{+}, \pi_{-}, \pi_{+}^{\prime}, \pi_{-}^{\prime}$ restricted to $L^{*}$. Here is such a theorem.

Theorem 5 Let $\pi_{\theta}$ (resp. $\pi_{\theta}^{\prime}$ ) be the irreducible admissible representation of $G L_{2}\left(k_{u}\right)$ (resp. $D_{u}^{*}$ ) associated to a character $\theta$ of $L^{*}$. Fix embeddings of $L^{*}$ in $G L_{2}\left(k_{u}\right)^{+}$, and $D_{u}^{*+}$ (in general there are two conjugacy classes of such embeddings). Let $\psi$ be a nontrivial additive character of $L$ trivial on $k_{u}$. The restriction of $\pi_{\theta}$ to $G L_{2}\left(k_{u}\right)^{+}$can be written as $\pi_{\theta}=\pi_{+} \oplus \pi_{-}$and the restriction of $\pi_{\theta}^{\prime}$ to $D_{u}^{*+}$ can be written as $\pi_{\theta}^{\prime}=\pi_{+}^{\prime} \oplus \pi_{-}^{\prime}$, such that a character $\chi$ of $L^{*}$ with 
$\left.\left(\chi \cdot \theta^{-1}\right)\right|_{k_{u}^{*}}=\omega_{L / k_{u}}$ appears in $\pi_{+}$if and only if $\epsilon\left(\theta \chi^{-1}, \psi\right)=\epsilon\left(\bar{\theta} \chi^{-1}, \psi\right)=1$, and appears in $\pi_{-}$if and only if $\epsilon\left(\theta \chi^{-1}, \psi\right)=\epsilon\left(\bar{\theta} \chi^{-1}, \psi\right)=-1$. Similarly, a character $\chi$ of $L^{*}$ with $\left.\left(\chi \cdot \theta^{-1}\right)\right|_{k_{u}^{*}}=\omega_{L / k_{u}}$ appears in $\pi_{+}^{\prime}$ if and only if $\epsilon\left(\theta \chi^{-1}, \psi\right)=1$, and $\epsilon\left(\bar{\theta} \chi^{-1}, \psi\right)=-1$, and appears in $\pi_{-}^{\prime}$ if and only if $\epsilon\left(\theta \chi^{-1}, \psi\right)=-1$, and $\epsilon\left(\bar{\theta} \chi^{-1}, \psi\right)=1$.

This theorem was proved in the odd residue characteristic case by this author in [P2]. We now prove this theorem in general by a global argument similar to the one in the earlier sections. The following lemma will play an important role in transferring information from a finite prime of a number field to an infinite prime.

Lemma 3 Let $k$ be a CM number field, and $u$ a place of $k$. Let $\theta: k_{u}^{*} \rightarrow \mathbb{C}^{*}$ be a character. Then there exists a Grössencharacter $\Theta: \mathbb{A}_{k}^{*} / k^{*} \rightarrow \mathbb{C}^{*}$ which is unramified at all the finite primes outside $u$, has $\theta$ as the local component at $u$, and whose local component at infinity is given by

$$
\Theta_{\infty}\left(z_{1}, \cdots, z_{r}\right)=z_{1}^{n_{1}} \cdots z_{k}^{n_{r}}, \quad \text { for } \quad\left|z_{i}\right|=1 \text {, }
$$

where $\left(n_{1}, \cdots, n_{r}\right)$ is any element of a certain subgroup of finite index in $\mathbb{Z}^{r}$.

Proof : Let $U_{k}=\prod_{v \in S_{f}} U_{v} \prod_{v \in S_{\infty}} \mathbb{S}^{1}$ be the maximal compact subgroup of $\mathbb{A}_{k}^{*}$. Define a character $\mu$ on $U_{k}$ by declaring its value on $U_{u}$ to be $\theta$ restricted to $U_{u}$, trivial on $U_{v}, v \neq u$, and given on $\left(z_{1}, \cdots, z_{r}\right) \in\left(\mathbb{S}^{1}\right)^{r}$ to be $z_{1}^{n_{1}} \cdots z_{r}^{n_{r}}$. Since $k^{*} \cap U_{k}=\mu_{k}$, the group of roots of unity in $k^{*},\left.\mu\right|_{k^{*} \cap U_{k}}$ is a character of finite order which can be assumed to be trivial by choosing the $r$-tuple of integers $\left(n_{1}, \cdots, n_{r}\right) \in \mathbb{Z}^{r}$ from a subgroup of finite index in $\mathbb{Z}^{r}$.

Thus we have a character, say $\Theta^{\prime}$, of the group $U_{k} /\left(k^{*} \cap U_{k}\right)$. Since, $U_{k} /\left(k^{*} \cap\right.$ $\left.U_{k}\right) \hookrightarrow \mathbb{A}_{k}^{*} / k^{*}$, and $U_{k} /\left(k^{*} \cap U_{k}\right)$ is a compact group, in particular closed, the character $\Theta^{\prime}$ can be extended to a Grössencharacter, say $\Theta^{\prime \prime}$, of $\mathbb{A}_{k}^{*} / k^{*}$. Observe that at the moment we have constructed a Grössencharacter $\Theta^{\prime \prime}$ of $\mathbb{A}_{k}^{*} / k^{*}$ which on $U_{u}$ is $\theta$ restricted to $U_{u}$. However, we still have the character $|\cdot|: \mathbb{A}_{k}^{*} / k^{*} \rightarrow \mathbb{C}^{*}$ given by $x \rightarrow|x|$, which is trivial on $U_{k}$, and takes the value $q_{u}^{-1}$ on $\pi_{u} \in k_{u}^{*} \subset \mathbb{A}_{k}^{*} / k^{*}$. Therefore for an appropriate choice of $s_{0} \in \mathbb{C}$, we can assume that the Grössencharacter $\Theta=\Theta^{\prime \prime}|\cdot|^{s_{0}}$ has $\theta$ as its local component at $u$, is unramified at all the other finite places, and has the desired behaviour at infinity. 
Proof of theorem 5: Using the theorem of Saito-Tunnell, we note that this theorem is equivalent to proving that for a character $\chi$ of $L^{*}$ appears in an irreducible component of $\pi_{\theta}$ restricted to $G L_{2}\left(k_{u}\right)^{+}$if and only if $\epsilon\left(\theta \chi^{-1}, \psi_{0}\right)$ as well as $\epsilon\left(\bar{\theta} \chi^{-1}, \psi_{0}\right)$ are independent of $\chi$; similarly for $D_{u}^{*+}$.

We choose a totally real number field $k$ and a place $u$ of $k$ such that the completion of $k$ at $u$ is $k_{u}$. Let $F$ be a quadratic extension of $k$ which is a CM field for which $F \otimes_{k} k_{u} \cong L$. Assume that $F$ is split at all the places of $k$ of residue characteristic 2 except $u$ (if $u$ is of residue characteristic 2). Let $D$ be a quaternion division algebra over $k$ containing $F$ for which $D \otimes_{k} k_{u} \cong D_{u}$, which is split at all the finite places of $k$ except $u$, and which remains a division algebra at all the infinite places of $k$. Such a choice of the triple $(k, F, D)$ is possible, as can be seen.

Let $\omega_{F / k}=\prod_{v} \omega_{v}$ be the quadratic character of $\mathbb{A}_{k}^{*} / k^{*}$ defining $F$. Define

$$
D^{*+}\left(\mathbb{A}_{k}\right)=\left\{g \in D^{*}\left(\mathbb{A}_{k}\right), g=\prod g_{v} \mid \omega_{v}\left(g_{v}\right)=1, \quad \forall \text { places } v \text { of } k\right\} \text {. }
$$

Let $D^{*+}(k)=D^{*} \cap D^{*+}\left(\mathbb{A}_{k}\right)$. Clearly $D^{*+}\left(\mathbb{A}_{k}\right)$ is an open subgroup of $D^{*}\left(\mathbb{A}_{k}\right)$ containing $D^{*+}(k)$.

Let $\pi$ be an automorphic representation of $D^{*}\left(\mathbb{A}_{k}\right)$ obtained from a character $\Theta: \mathbb{A}_{F}^{*} / F^{*} \rightarrow \mathbb{C}^{*}$ which is unramified at all the finite places of $F$ except $u$, and is $\theta$ on $F_{u}^{*}$; this is possible by lemma 3. Let $\pi^{+}=\otimes \pi_{w}^{+}$be an irreducible automorphic representation of $D^{*+}\left(\mathbb{A}_{k}\right)$ contained in $\pi$ with $\pi_{u}^{+}=\pi_{+}$.

Let $T$ be the torus inside $D^{*}$ defined by $F$. By lemma 1 , there exists a continuous character $\Omega=\otimes \Omega_{v}$ of $T(k) \backslash T\left(\mathbb{A}_{k}\right)$ with $\Omega_{u}=\chi$ whose restriction to the center of $D\left(\mathbb{A}_{k}\right)^{*}$ is the same as the central character of $\pi$, and for which there exists a function $e$ in $\pi^{+}$such that the integral

$$
\int_{T(k) \mathbb{A}_{k}^{*} \backslash T\left(\mathbb{A}_{k}\right)} e(t) \Omega^{-1}(t) d t
$$

is nonzero. By the theorem of Waldspurger, if $\Pi=B C(\pi)$ denotes the base change of $\pi$ to $G L_{2}\left(\mathbb{A}_{F}\right)$,

$$
L\left(\frac{1}{2}, \Pi \otimes \Omega^{-1}\right) \neq 0 .
$$

Notice that

$$
\Pi=B C(\pi)=\operatorname{Ps}(\Theta \oplus \bar{\Theta}),
$$


where $\bar{\Theta}$ denotes the conjugate of $\Theta$ under the nontrivial element of the Galois group of $F$ over $k$. It follows that,

$$
L\left(s, \Pi \otimes \Omega^{-1}\right)=L_{F}\left(s, \Theta \cdot \Omega^{-1}\right) L_{F}\left(s, \bar{\Theta} \cdot \Omega^{-1}\right) .
$$

By the condition on the central characters, one sees that $\operatorname{Ind}_{F}^{k}\left(\Theta \cdot \Omega^{-1}\right)$ is a selfdual representation, and hence the functional equation for its $L$-function relates itself. Therefore, since

$$
L_{F}\left(s, \Theta \cdot \Omega^{-1}\right)=L_{k}\left(s, \operatorname{Ind}_{F}^{k}\left(\Theta \cdot \Omega^{-1}\right)\right),
$$

the functional equation for $L_{F}\left(s, \Theta \cdot \Omega^{-1}\right)$ relates itself; similarly, the functional equation for $L_{F}\left(s, \bar{\Theta} \cdot \Omega^{-1}\right)$ relates itself.

As $L\left(\frac{1}{2}, \Pi \otimes \Omega^{-1}\right) \neq 0$, so also are $L_{F}\left(\frac{1}{2}, \Theta \cdot \Omega^{-1}\right)$ and $L_{F}\left(\frac{1}{2}, \bar{\Theta} \cdot \Omega^{-1}\right)$, and therefore, in particular, the sign in their functional equations is 1 :

$$
\begin{aligned}
& \epsilon_{F}\left(\frac{1}{2}, \Theta \cdot \Omega^{-1}\right)=1 \\
& \epsilon_{F}\left(\frac{1}{2}, \bar{\Theta} \cdot \Omega^{-1}\right)=1 .
\end{aligned}
$$

The global epsilon factor is the product of the local ones:

$$
\epsilon_{F}\left(\frac{1}{2}, \Theta \cdot \Omega^{-1}\right)=\prod_{w} \epsilon_{w}\left(\frac{1}{2}, \Theta_{w} \cdot \Omega_{w}^{-1}\right) .
$$

We analyse the local epsilon factors at the various primes of $F$, and thereby prove the theorem, in following steps.

1. For places $u$ of $k$ which split into two places in $F$, say $w_{1}$ and $w_{2}$, we have

$$
\epsilon_{w_{1}}\left(\frac{1}{2}, \Theta_{w_{1}} \cdot \Omega_{w_{1}}^{-1}\right) \cdot \epsilon_{w_{2}}\left(\frac{1}{2}, \Theta_{w_{2}} \cdot \Omega_{w_{2}}^{-1}\right)=1 .
$$

This follows from the following general relation:

$$
\epsilon\left(\frac{1}{2}, \chi, \psi(x)\right) \cdot \epsilon\left(\frac{1}{2}, \chi^{-1}, \psi(-x)\right)=1
$$

which is what we have by the condition on the central character of $\pi$ (which is $\left.\Theta\right|_{\mathbb{A}_{k}^{*}} \cdot \omega_{F / k}$ ) being the same as the character $\Omega$ restricted to $\mathbb{A}_{k}^{*}$; we also need to note that the additive character of $\mathbb{A}_{F}$ with respect to which we are calculating the epsilon factor is trivial on $\mathbb{A}_{k}$, so is of the form $(\psi(x), \psi(-x))$ at a prime $w$ of $k$ which splits in $F$. 
2. For places $w$ of $F$ which are inert over the corresponding place $v$ in $k$, we note that since by the choice of $\Omega$, the $\Omega$-period integral is nonzero, we have local $\Omega_{w}$-invariant forms on $\pi_{w}^{+}$at all places. Since $\Theta$ is unramified at all finite places away from $u$, and thus is invariant under $\operatorname{Gal}(F / k)$, at such places, the representation $\pi_{w}$ is, up to a twist, of the form $\operatorname{Ps}(1, \omega)$ where $\omega$ is the quadratic character defining the extension $F_{w}$ of $k_{v}$. The next lemma now implies that

$$
\epsilon_{w}\left(\frac{1}{2}, \Theta_{w} \cdot \Omega_{w}^{-1}\right)=1
$$

at all finite places $w$ of $F$ inert over $k, w \neq u$.

3. The epsilon factor, $\epsilon_{w}\left(\frac{1}{2}, \Theta_{w} \cdot \Omega_{w}^{-1}\right)$ is -1 at any infinite place $w$ as $D$ remains a division algebra at such places. This once again follows because the period integral is nonzero, and hence there is an invariant linear form at every infinite place, and then appealing to the Archimedean analogue of our theorem which easily follows from the information given in section 5 .

4. Assume that $D_{u}=M_{2}\left(k_{u}\right)$. In this case, $D$ remains a division algebra at exactly the infinite primes, so they are even in number. We know that the local epsilon factor at all finite places of $F$ except $u$ is 1 , and is -1 at the even number of infinite places. Since the product of the local epsilon factors at all the places of $F$ is 1 , the epsilon factor $\epsilon_{u}\left(\frac{1}{2}, \Theta_{u} \cdot \Omega_{u}^{-1}\right)$ at the desired place $u$ is 1 .

5. Assume $D$ is ramified at $u$ and at all the infinite places, which will now be odd in number. In this case the local epsilon factor is 1 at all finite places except $u$, is -1 at all infinite places which are odd in number; therefore, the epsilon factor $\epsilon_{u}\left(\frac{1}{2}, \Theta_{u} \cdot \Omega_{u}^{-1}\right)=-1$ in this case, completing the proof of the theorem.

Lemma 4 Let $L$ be a quadratic extension of a local field $k_{u}$ of odd residue characteristic. Then the principal series representation $\pi=\operatorname{Ps}\left(1, \omega_{L / k_{u}}\right)$ splits into two irreducible representations $\pi=\pi_{+} \oplus \pi_{-}$when restricted to $G L_{2}\left(k_{u}\right)^{+}=$ $\left\{x \in G L_{2}\left(k_{u}\right) \mid \operatorname{det}(x) \in N L^{*}\right\}$, where $N L^{*}$ is the subgroup of $k_{u}^{*}$ of index 2 consisting of norms from $L^{*}$, such that a character $\chi$ of $L^{*}$ with $\left.\chi\right|_{k_{u}{ }^{*}}=\omega_{L / k_{u}}$ appears in $\pi_{+}$if and only if $\epsilon(\chi)=1$, and appears in $\pi_{-}$if and only if $\epsilon(\chi)=-1$. 
Proof : The following was proved in lemma 3.1 of [P2] by direct calculation in the odd residue characteristic:

$$
\epsilon\left(\omega_{L / k_{u}}, \psi\right) \frac{\omega_{L / k_{u}}\left(\frac{x-\bar{x}}{x_{0}-\bar{x}_{0}}\right)}{\left|\frac{(x-\bar{x})^{2}}{x \bar{x}}\right|_{k_{u}}^{1 / 2}}=\sum_{\substack{\epsilon_{L}\left(\chi, \psi_{0}\right)=\left.1 \\ \chi\right|_{k_{u}^{*}}=\omega_{L / k_{u}}}} \chi(x) .
$$

Here $\psi$ is a nontrivial character of $k, x_{0}$ an element of $L^{*}$ whose trace to $k_{u}$ is zero, and $\psi_{0}$ is an additive character on $L$ defined by $\psi_{0}(x)=\psi\left(\operatorname{tr}\left[-x x_{0} / 2\right]\right)$, and, where, as usual, the summation (on the right) is by partial sums over all characters of $L^{*}$ of conductor $\leq n$.

It has been proved by Langlands in [L], Lemma 7.19 (the lemma with an 'embarassing' proof), that the character $\Theta_{\pi_{+}}$of $\pi_{+}$is given by

$$
\Theta_{\pi_{+}}(x)=\epsilon\left(\omega_{L / k_{u}}, \psi\right) \frac{\omega_{L / k_{u}}\left(\frac{x-\bar{x}}{x_{0}-\bar{x}_{0}}\right)}{\left|\frac{(x-\bar{x})^{2}}{x \bar{x}}\right|_{k_{u}}^{1 / 2}} .
$$

These two results combine to prove lemma 4.

Remark : We have stated and proved the lemma only in the odd residue characteristic; this is enough for our purposes to prove the theorem 5 in all residue characteristics. The statement of the theorem includes this lemma as a particular case, and therefore after we have proved the theorem 5 using this lemma in odd residue characteristic, we get a proof of lemma 4 in all residue characteristics by global method! Also, since the lemma of Langlands is available in all residue characteristics, we get a proof of the following lemma proved by this author only in odd residue charateristic earlier in [P2].

Lemma 5 Let $L$ be a quadratic extension of a local field $k_{u}$. Fix a nontrivial character $\psi$ of $k_{u}$, and an element $x_{0}$ in $L^{*}$ whose trace to $k_{u}$ is zero. Define an additive character $\psi_{0}$ on $L$ by $\psi_{0}(x)=\psi\left(\operatorname{tr}\left[-x x_{0} / 2\right]\right)$. Then,

$$
\epsilon\left(\omega_{L / k_{u}}, \psi\right) \frac{\omega_{L / k_{u}}\left(\frac{x-\bar{x}}{x_{0}-\bar{x}_{0}}\right)}{\left|\frac{(x-\bar{x})^{2}}{x \bar{x}}\right|_{k_{u}}^{1 / 2}}=\sum_{\begin{array}{l}
\epsilon(\chi, \psi)=1 \\
\left.\chi\right|_{k_{u}^{*}}=\omega_{L / k_{u}}
\end{array}} \chi(x)
$$


where as usual, the summation on the right is by partial sums over all characters of $L^{*}$ of conductor $\leq n$.

Remark : Given a quadratic extension $L$ of a local field $k_{u}$, the group $L^{1}$ of norm 1 elements of $L^{*}$ can be embedded in $S L_{2}\left(k_{u}\right)$. The two fold metaplectic cover $M p_{2}\left(k_{u}\right)$ of $S L_{2}\left(k_{u}\right)$ splits over $L^{1}$ (however, the splitting depends on the choice of a character $\chi_{0}$ of $L^{*}$ such that $\left.\chi_{0} \mid k_{u}^{*}=\omega_{L / k_{u}}\right)$. Thus one can speak of the restriction of the Weil representation $\omega_{\psi}$ of $M p_{2}\left(k_{u}\right)$, associated to a nontrivial additive character $\psi$ of $k_{u}$, to $L^{1}$. Characters $\mu$ of $L^{1}$ can be identified to characters $\chi$ of $L^{*}$ with $\chi \mid k_{u}^{*}=\omega_{L / k_{u}}$ by $\chi(x)=\mu(x / \bar{x}) \chi_{0}(x)$. It is a theorem of C. Moen, cf. [M], in odd residue characteristic, proved by J. Rogawski, cf. [Ro], in general, that a character $\chi$ of $L^{*}$ with $\chi \mid k_{u}^{*}=\omega_{L / k_{u}}$ appears in $\omega_{\psi}$ if and only if $\epsilon(\chi, \psi)=1$. Thus lemma 4 implies that the Weil representation $\omega_{\psi}$ restricted to $L^{1}$ is closely related to a component of a reducible principal series. What lies behind this phenomenon- relating character of a linear and a non-linear group- is not clear to this author.

\section{Symmetric and exterior squares}

The results in [P1] on the trilinear forms were refined in [P2] in the case when $\pi_{1}=\pi_{2}=\pi$. In this case,

$$
\pi \otimes \pi=\operatorname{Sym}^{2}(\pi) \oplus \wedge^{2}(\pi)
$$

Similarly for the corresponding Galois representations,

$$
\sigma_{\pi} \otimes \sigma_{\pi}=\operatorname{Sym}^{2}\left(\sigma_{\pi}\right) \oplus \wedge^{2}\left(\sigma_{\pi}\right) .
$$

Here is the theorem about symmetric squares proved in [P2] in the odd residue characteristic, now proved in general. It should also be pointed out that the methods of the Weil representation employed in [P2] could give results about symmetric and exterior square only for $G L_{2}$, and not for the representations of invertible elements of a division algebra!

Theorem 6 Let $D_{u}$ be a quaternion algebra over $k_{u}$. Let $\epsilon_{D}$ be 1 if $D_{u}=$ $M_{2}\left(k_{u}\right)$, and $\epsilon_{D}=-1$ otherwise. For irreducible admissible representations $\pi$ and $\pi^{\prime}$ of $D_{u}^{*}$ (which will be assumed to be infinite dimensional if $D_{u}^{*}=$ $\left.G L_{2}\left(k_{u}\right)\right)$ with $\omega_{\pi}^{2} \omega_{\pi^{\prime}}=1, \operatorname{Sym}^{2}(\pi) \otimes \pi^{\prime}$ has a $D_{u}^{*}$-invariant linear form if and 
only if $\epsilon\left(\operatorname{Sym}^{2}\left(\sigma_{\pi}\right) \otimes \sigma_{\pi^{\prime}}\right)=\omega_{\pi}(-1)$, and $\epsilon\left(\wedge^{2}\left(\sigma_{\pi}\right) \otimes \sigma_{\pi^{\prime}}\right)=\epsilon_{D} \omega_{\pi}(-1)$. The representation $\wedge^{2}(\pi) \otimes \pi^{\prime}$ has a $G L_{2}\left(k_{u}\right)$-invariant linear form if and only if $\epsilon\left(\operatorname{Sym}^{2}\left(\sigma_{\pi}\right) \otimes \sigma_{\pi^{\prime}}\right)=-\omega_{\pi}(-1)$, and $\epsilon\left(\wedge^{2}\left(\sigma_{\pi}\right) \otimes \sigma_{\pi^{\prime}}\right)=-\epsilon_{D} \omega_{\pi}(-1)$.

Proof : Let $k$ be a totally real number field with a finite place $u$ where the completion of $k$ is $k_{u}$. Let $D$ be a quaternion division algebra over $k$ for which $D \otimes_{k} k_{u} \cong D_{u}$, which is split at all the other finite primes, and which remains a division algebra at all the places at infinity. Such a choice of the pair $(k, D)$ exists.

Let $\Pi$ be an irreducible automorphic representation of $D^{*}\left(\mathbb{A}_{k}\right)$ with $\pi$ as the local component at $u$, unramified at all the other finite places of $k$, and certain representations at infinity. By lemma 1 , there exists a representation $\Pi^{\prime}$ of $D^{*}\left(\mathbb{A}_{k}\right)$ with local components $\pi^{\prime}$ at $u$, functions $f_{1}, f_{2} \in \Pi$, and $f^{\prime} \in \Pi^{\prime}$ such that

$$
\int_{D^{*} \mathbb{A}_{k}^{*} \backslash D^{*}\left(\mathbb{A}_{k}\right)} f_{1} f_{2} f^{\prime} d g \neq 0
$$

By the theorem of Harris-Kudla, $L\left(\frac{1}{2}, \Pi \times \Pi \times \Pi^{\prime}\right) \neq 0$. The proof proceeds, once again, using the factorisation of $L$-functions,

$$
L\left(s, \Pi \times \Pi \times \Pi^{\prime}\right)=L\left(s, \operatorname{Sym}^{2}(\Pi) \times \Pi^{\prime}\right) L\left(s, \wedge^{2}(\Pi) \times \Pi^{\prime}\right),
$$

all of which are known to be analytic at $1 / 2$. Therefore if $L\left(\frac{1}{2}, \Pi \times \Pi \times \Pi^{\prime}\right) \neq 0$, then both $L\left(\frac{1}{2}, \operatorname{Sym}^{2}(\Pi) \times \Pi^{\prime}\right)$ and $L\left(\frac{1}{2}, \wedge^{2}(\Pi) \times \Pi^{\prime}\right)$ are nonzero. Since we are once again dealing with self-dual representations, this forces global epsilon factors to be 1 :

$$
\begin{aligned}
\epsilon\left(\frac{1}{2}, \operatorname{Sym}^{2}(\Pi) \times \Pi^{\prime}\right) & =1 \\
\epsilon\left(\frac{1}{2}, \wedge^{2}(\Pi) \times \Pi^{\prime}\right) & =1 .
\end{aligned}
$$

We have the factorisation of epsilon factors,

$$
\epsilon\left(\frac{1}{2}, \operatorname{Sym}^{2}(\Pi) \times \Pi^{\prime}\right)=\prod_{v} \epsilon\left(\frac{1}{2}, \operatorname{Sym}^{2}\left(\Pi_{v}\right) \times \Pi_{v}^{\prime}\right) .
$$

Observe that the linear form

$$
f_{1} \otimes f_{2} \otimes f^{\prime} \longrightarrow \int_{D^{*} \mathbb{A}_{k}^{*} \backslash D^{*}\left(\mathbb{A}_{k}\right)} f_{1} f_{2} f^{\prime} d g
$$


defines a $D^{*}\left(\mathbb{A}_{k}\right)$-invariant linear form on $\Pi \otimes \Pi \otimes \Pi^{\prime}$ which is symmetric in the first 2 variables. By the local uniqueness of the trilinear form, the invariant form on $\Pi_{w} \otimes \Pi_{w} \otimes \Pi_{w}^{\prime}$ is either symmetric or skew-symmetric in the first 2 variables. By generalities about group representations, it follows that the set of places $w$ of $k$ for which the invariant form on $\Pi_{w} \otimes \Pi_{w} \otimes \Pi_{w}^{\prime}$ is skew-symmetric is even. By choice, $\Pi_{w}$ is an unramified principal series at all finite places $w \neq u$; in particular, we have the condition on the central character $\omega_{\Pi_{w}}(-1)=1$. By the following lemma from [P3], the local invariant forms on $\Pi_{w} \otimes \Pi_{w} \otimes \Pi_{w}^{\prime}$ at all finite places $w \neq u$ are symmetric.

Lemma 6 Suppose $\pi_{v}$ is a principal series representation of $G L_{2}\left(k_{v}\right)$. Then for an irreducible admissible representation $\pi_{v}^{\prime}$ of $G L_{2}\left(k_{v}\right), \operatorname{Sym}^{2}\left(\pi_{v}\right) \otimes \pi_{v}^{\prime}$ has a $G L_{2}\left(k_{v}\right)$-invariant linear form if and only if $\omega_{\pi_{v}}(-1)=1$.

Theorem 6 now follows from the following lemma at infinity whose simple proof will be omitted.

Lemma 7 Let $\mathbb{H}$ be the quaternion division algebra over $\mathbb{R}$. For irreducible representations $\pi$ and $\pi^{\prime}$ of $\mathbb{H}^{*}$ with $\omega_{\pi}^{2} \omega_{\pi^{\prime}}=1$, $\operatorname{Sym}^{2}(\pi) \otimes \pi^{\prime}$ has a $\mathbb{H}^{*}$-invariant linear form if and only if $\epsilon\left(\operatorname{Sym}^{2}\left(\sigma_{\pi}\right) \otimes \sigma_{\pi^{\prime}}\right)=\omega_{\pi}(-1)$, and $\epsilon\left(\wedge^{2}\left(\sigma_{\pi}\right) \otimes \sigma_{\pi^{\prime}}\right)=$ $-\omega_{\pi}(-1)$. The representation $\wedge^{2}(\pi) \otimes \pi^{\prime}$ has a $\mathbb{H}^{*}$-invariant linear form if and only if $\epsilon\left(\operatorname{Sym}^{2}\left(\sigma_{\pi}\right) \otimes \sigma_{\pi^{\prime}}\right)=-\omega_{\pi}(-1)$, and $\epsilon\left(\wedge^{2}\left(\sigma_{\pi}\right) \otimes \sigma_{\pi^{\prime}}\right)=\omega_{\pi}(-1)$.

\section{Archimedean Case}

The results in this paper have eventually depended on similar but much simpler questions for the Archimedean field (which can be assumed to be $\mathbb{R}$ ). For this, we fix some notation and recall some standard facts without going into the proofs of the Archimdean lemmas used in this paper. We will let $\mathbb{H}$ denote the quaternion division algebra over $\mathbb{R}$.

The Weil group $W_{\mathbb{C} / \mathbb{R}}$ of $\mathbb{R}$ is the normaliser of $\mathbb{C}^{*}$ in $\mathbb{H}^{*}$ and sits in the exact sequence :

$$
0 \rightarrow \mathbb{C}^{*} \rightarrow W_{\mathbb{C} / \mathbb{R}} \rightarrow \mathbb{Z} / 2 \mathbb{Z} \rightarrow 0 .
$$

Let $\psi(x)=\exp (2 \pi i x)$ be the character of $\mathbb{R}$, and $\psi_{0}(z)=\psi\left(\operatorname{tr}_{\mathbb{C} / \mathbb{R}} i z\right)$ be a character of $\mathbb{C}$ which is trivial on $\mathbb{R}$. Then for integers $n, m$, and complex number $s$, we have, 


$$
\begin{array}{rlrl}
\epsilon\left(z^{n} \bar{z}^{m}|z|^{s}, \psi_{0}\right) & =(-1)^{n-m} & \text { if } n \geq m \\
& = & 1 & \text { if } n<m .
\end{array}
$$

For $m \geq 0$, let $\sigma_{m}$ be the two dimensional representation $\operatorname{lnd}_{\mathbb{C}^{*} / \mathbb{R}}^{W_{\mathbb{R}}}(z /|z|)^{m}$ of $W_{\mathbb{C} / \mathbb{R}}$. We have,

$$
\epsilon\left(\sigma_{m}, \psi\right)=i^{m+1} \quad \text { for } \quad m \geq 0 \text {. }
$$

It follows that,

$$
\begin{aligned}
\epsilon\left(\sigma_{m} \otimes \sigma_{n}, \psi\right) & =(-1)^{m+1} \quad \text { for } \quad m \geq n \\
& =(-1)^{n+1} \quad \text { for } \quad n \geq m
\end{aligned}
$$

Under the Langlands correspondence, the discrete series representation $D_{m}$ of $G L_{2}(\mathbb{R})$ for $m \geq 2$, which has trivial central character restricted to $\mathbb{R}^{*+}$, corresponds to the representation $\sigma_{m-1}$ of the Weil group $W_{\mathbb{C} / \mathbb{R}}$. The corresponding representation of $\mathbb{H}^{*} / \mathbb{R}^{*+}$, to be denoted by $F_{m-2}$, is of dimension $m-1$, and of highest weight $z^{m-2} /|z|^{m-2}$.

The proof of the following lemma is standard, and will therefore be omitted. Using the information on epsilon factors given in this section, this is then easily seen to be equivalent to lemma 7 .

Lemma 8 Let $F_{n}$ denote the irreducible representation of $\mathbb{H}^{*} / \mathbb{R}^{*+}$ of highest weight $n$. Then,

$$
\begin{aligned}
\operatorname{Sym}^{2}\left(F_{n}\right) & =F_{2 n} \oplus F_{2 n-4} \oplus \cdots \\
\Lambda^{2} F_{n} & =F_{2 n-2} \oplus F_{2 n-6} \oplus \cdots
\end{aligned}
$$

Remark : For the purposes of this paper, it is curious to note that we can assume that the groups are compact at infinity, and therefore can deduce theorems about (discrete series representations) of noncompact real groups from that of its compact form via the global theorems!

\section{Epilogue}

Results in this paper were proved by using the Burger-Sarnak principle after applying the theorem of Harris-Kudla or Waldspurger. These results enabled us to prove nonvanishing of central critical value of certain $L$-functions which played a crucial role in our applications. There is a very large literature on the 
nonvanishing results of this kind, but we do not detail them here. However, we take the opportunity to state the following general questions about $L$-functions which we have answered in this paper in some of the cases we studied here, and which might be amenable to do by similar methods.

\section{Questions:}

1. Given a cuspidal automorphic representation $\Pi$ of $G L_{n}\left(\mathbb{A}_{k}\right)$, and an integer $m \leq n$, is there a cuspidal automorphic representation $\Pi^{\prime}$ on $G L_{m}\left(\mathbb{A}_{k}\right)$ with prescribed discrete series behaviour at finitely many places of $k$, and with trivial central character for $\Pi \times \Pi^{\prime}$, such that

$$
L\left(\frac{1}{2}, \Pi \times \Pi^{\prime}\right) \neq 0
$$

2. Given a cuspidal automorphic representation $\Pi$ of $G L_{n}\left(\mathbb{A}_{k}\right)$ which is selfdual up to a twist, and an integer $m \leq n$, is there a cuspidal automorphic representation $\Pi^{\prime}$ on $G L_{m}\left(\mathbb{A}_{k}\right)$ which is self-dual up to a twist, has prescribed discrete series behaviour at finitely many places of $k$, such that $\Pi \times \Pi^{\prime}$ is self-dual, and such that

$$
L\left(\frac{1}{2}, \Pi \times \Pi^{\prime}\right) \neq 0 .
$$

Acknowledgement : The author thanks T.N. Venkataramana for discussions on the Burger-Sarnak 'principle' on several occasions, and also thanks U.K. Anandavardhanan for some helpful discussions.

\section{References}

[BS] M.Burger and P. Sarnak. Ramanujan duals. II. Invent. Math. 106 (1991), no. $1,1-11$.

[GP] B. Gross and D. Prasad, On the decomposition of a representation of $\mathrm{SO}_{n}$ when restricted to $\mathrm{SO}_{n-1}$. Canad. J. Math. 44 (1992), no. 5, 974-1002.

[HK] M. Harris and S. Kudla, On a conjecture of $H$. Jacquet. Contributions to Automorphic forms, Geometry, and Number Theory, 355-371; Johns Hopkins Univ. Press, Baltimore (2004); edited by H. Hida, D. Ramakrishnan, and F. Shahidi. 
[HL] M. Harris and J.-S. Li, A Lefschetz property for subvarieties of Shimura varieties. J. Algebraic Geom. 7 (1998), no. 1, 77-122.

[L] R. Langlands, Base Change for $G L(2)$, Annals of Maths studies, Princeton University Press, Princeton (1980).

[M] C. Moen, The dual pair $(\mathrm{U}(1), \mathrm{U}(1))$ over a p-adic field. Pacific J. Math. 158 (1993), no. 2, 365-386.

[P1] D. Prasad, Trilinear forms for representations of $G L(2)$ and local $\epsilon-$ factors. Compositio Math. 75 (1990), no. 1, 1-46.

[P2] D. Prasad, On an extension of a theorem of Tunnell, Compositio 94 (1994), 19-28.

[P3] D.Prasad, Some applications of seesaw duality to branching laws, Math. Ann. 304 (1996), 1-20.

[Ra] D. Ramakrishnan. Modularity of the Rankin-Selberg L-series, and multiplicity one for SL(2). Ann. of Math. (2) 152 (2000), no. 1, 45-111.

[Ro] J. Rogawski. The multiplicity formula for A-packets. The zeta functions of Picard modular surfaces, 395-419, Univ. Montral, Montreal, QC, 1992.

[Sa] H. Saito. On Tunnell's formula for characters of GL(2). Compositio Math. 85 (1993), no. 1, 99-108.

[Tu] J. Tunnell. Local $\epsilon$-factors and characters of GL(2). Amer. J. Math. 105 (1983), no. 6, 1277-1307.

[Wa] J.-L. Waldspurger. Sur les valeurs de certaines fonctions $L$ automorphes en leur centre de symtrie. Compositio Math. 54 (1985), no. 2, 173-242.

School of Mathematics, Tata Institute of Fundamental Research,

Colaba, Mumbai-400005, INDIA

Email: dprasad@math.tifr.res.in 